\title{
IncRNA NORAD is consistently detected in breastmilk exosomes and its expression is downregulated in mothers of preterm infants
}

\author{
NIKI MOURTZI $^{1}$, TANIA SIAHANIDOU ${ }^{2}$, MARGARITIS TSIFINTARIS ${ }^{3}$, EIRINI KARAMICHALI ${ }^{4}$, \\ ANDRONIKI TASIOPOULOU ${ }^{3}$, AMALIA SERTEDAKI ${ }^{1}$, MARGARITA PESMATZOGLOU ${ }^{2}$, \\ ANASTASIA KAPETANAKI ${ }^{5}$, GEORGE LIOSIS ${ }^{5}$, GEORGE BALTATZIS ${ }^{6}$, DIMITRIOS VLACHAKIS ${ }^{7,8}$, \\ GEORGE P. CHROUSOS ${ }^{8}$ and ANTONIS GIANNAKAKIS ${ }^{3,8}$
}

${ }^{1}$ Laboratory of Molecular Endocrinology, Choremeio Research Center, First Department of Pediatrics, Children's Hospital 'Aghia Sophia', School of Medicine, National and Kapodistrian University of Athens;

${ }^{2}$ First Department of Pediatrics, 'Aghia Sophia' Children's Hospital, School of Medicine, National and Kapodistrian University of Athens, 11527 Athens; ${ }^{3}$ Laboratory of Gene Expression,

Molecular Diagnostics and Modern Therapeutics, Department of Molecular Biology and Genetics,

Democritus University of Thrace, 68100 Alexandroupolis; ${ }^{4}$ Laboratory of Molecular Virology,

Hellenic Pasteur Institute; ${ }^{5}$ General and Maternity Hospital 'Helena Venizelou', 11521 Athens;

${ }^{6}$ First Department of Pathology, School of Health Sciences, School of Medicine,

National and Kapodistrian University of Athens, 11527 Athens; ${ }^{7}$ Laboratory of Genetics, Department of Biotechnology,

School of Applied Biology and Biotechnology, Agricultural University of Athens, 11855 Athens;

${ }^{8}$ University Research Institute of Maternal and Child Health and Precision Medicine, and

UNESCO Chair on Adolescent Health Care, National and Kapodistrian University of Athens, 11527 Athens, Greece

Received July 12, 2021; Accepted September 27, 2021

DOI: $10.3892 / \mathrm{ijmm} .2021 .5049$

\begin{abstract}
Breast milk is the ideal food for infants and undoubtedly has immediate and long-term benefits. Breast milk contains extracellular vesicles (EVs) i.e., exosomes secreted by maternal breast cells. Exosomes carry genetic material, such as long non-coding RNAs (lncRNAs), which possibly participate in cell-to-cell communications, as they are known to regulate critical gene pathways. The aim of the present study was to screen human breastmilk exosomes for their lncRNA cargo and to examine exosomal lncRNA levels associated with milk obtained from mothers that gave birth
\end{abstract}

Correspondence to: Dr Antonis Giannakakis, Laboratory of Gene Expression, Molecular Diagnostics and Modern Therapeutics, Department of Molecular Biology and Genetics, Democritus University of Thrace, Draganas 10, 68100 Alexandroupolis, Greece E-mail: antgian@mbg.duth.gr

Professor George P. Chrousos, University Research Institute of Maternal and Child Health and Precision Medicine, and UNESCO Chair on Adolescent Health Care, National and Kapodistrian University of Athens, 8 Thivon and Livadias Street, 11527 Athens, Greece

E-mail: chrousos@gmail.com

Key words: breastmilk, exosomes, long non-coding RNAs, preterm birth, non-coding RNA activated at DNA damage at term or prematurely ( $<37$ weeks of gestation). Samples were collected at 3 weeks postpartum from 20 healthy, breastfeeding mothers; 10 mothers had given birth at full-term and 10 mothers preterm. Exosomal RNA was extracted from all samples and the expression of 88 distinct lncRNAs was determined using reverse transcription-quantitative PCR. A total of 13 lncRNAs were detected in $\geq 85 \%$ of the samples, while 31 were detected in $\geq 50 \%$ of the samples. Differential expression analysis of the lncRNAs between the two groups revealed $\geq 2$-fold differences, with generally higher lncRNA concentrations found in the milk of the mothers that gave birth at term compared with those that gave birth preterm. Among these, the non-coding RNA activated at DNA damage $(N O R A D)$ was prominently detected in both groups, and its expression was significantly downregulated in the breast milk exosomes of mothers who delivered preterm. On the whole, the present study demonstrates that breast milk lncRNAs may be important factors of normal early human development. Collectively, the presence of IncRNAs in human breast milk may explain the consistent inability of researchers to fully 'humanize' animal milk.

\section{Introduction}

Preterm birth affects $>15$ million infants worldwide (1). These infants are at a significantly increased risk of developing acute adverse health outcomes, such as life-long metabolic (2) and immune health complications (3). Breast milk confers significant benefits to infant health, including a decreased 
risk of sudden infant death syndrome, sepsis and necrotizing enterocolitis (4). It has also been associated with improved neurodevelopmental outcomes and protection against metabolic diseases, including obesity and diabetes mellitus type 1 and 2 during the later stages of life $(5,6)$. The latter is further illustrated by recent studies demonstrating the modulatory effects of breastfeeding on the expression of genes, such as the fat mass and obesity-associated (FTO) gene, which interacts with mRNAs/long non-coding RNAs (lncRNAs) to influence body mass index and fat tissue growth (7-9). Human milk composition is unique; however, it is influenced by maternal factors, including stress, nutrition, presence of inflammation and preterm birth $(10,11)$. Apart from nutrients, human breast milk contains a heterogeneous array of non-nutritive components, including enzymes, hormones, growth factors, immune cells, stem cells, bacteria, antibodies, cytokines, antimicrobial peptides and extracellular vesicles (EVs), that may play significant roles in the development of infants $(12,13)$.

EVs comprise a vast spectrum of microparticles and based on the corresponding size range and biogenesis, can be broadly classified into three distinct classes: Microvesicles, exosomes and apoptotic bodies (14). Microvesicles have a diameter ranging from $50 \mathrm{~nm}$ to $1 \mu \mathrm{m}$ and are released by cell membrane budding. Exosomes are particles with a diameter ranging from $30-150 \mathrm{~nm}$ and are derived via a targeted mechanism from the cell endocytic compartment through the formation of multivesicular bodies (MVBs) (15). Exosomes are small lipid-bound vesicles released from all cells into the extracellular space or biological fluids (16). They are highly heterogeneous as regards their size, content and function to recipient cells and cells of origin, and they can induce a repertoire of biological responses. Exosomes are considered as an integral part of the senescence-associated secretory phenotype (17) and depending on their cargo or surface composition, they may function as specific signals/mediators of systemic stress between cells or between tissues (14). Breast milk is a biofluid enriched in exosomes. Previous studies have indicated the protective role of human milk-derived exosomes, focusing on their role against necrotizing enterocolitis in preterm infants $(9,18)$. Furthermore, exosomes in breast milk may be central epigenetic regulators as regards the expression of developmental genes, such as FTO, insulin (INS) and insulin like growth factor $1(I G F 1)(9)$.

A number of studies have suggested that exosomes harbor a variety of active or non-autonomous biomolecules, including proteins, lipids, DNA, mRNAs, microRNAs (miRNAs/miRs) and lncRNAs. Thus, owing to their unique potential to group multiple signals together, exosomes constitute an alternative and largely unexplored mode of communication between neighboring and distant cells, differing from the conventional hormone-mediated mechanisms $(14,15,19,20)$. Likewise, regulatory non-coding RNAs (ncRNAs) have already been established as an appealing new source of novel 'genetic hormones' and biomarkers, with an increased sensitivity and specificity for an unprecedented range of diseases, conditions or cell states (20). There is a growing body of literature that recognizes the crucial roles of lncRNAs in gene regulation as they can interact with proteins, DNA and RNA, and modulate mRNA expression, chromatin function and signaling pathways (21). Of note, the RNA cargo in exosomes does not simply reflect the tissue and cell state, nor does it represent the RNA composition of the cell of origin, but rather a selective sorting and loading of specific RNAs into EVs. IncRNAs are at the forefront of both basic biological and clinical research due to their immense predictive value as novel biomarkers in precision medicine, as well as their significant therapeutic potential, given that lncRNAs are considered easier to be targeted for disease prevention and therapy, compared to protein-coding genes. Previous studies have revealed the presence of miRNAs (22) and lncRNAs in EVs in breast milk $(12,23)$. Karlsson et al (12) detected exosomal lncRNAs in breast milk, including colorectal neoplasia differentially expressed $(C R N D E)$ gene, differentiation antagonizing non-protein coding RNA (DANCR), growth arrest-specific 5 (GAS5), steroid receptor RNA activator 1 (SRA1) and ZNFX1 antisense RNA 1 (ZFASI), that may represent epigenetic regulators involved in child development.

The present study demonstrates that exosomes circulating in human breast milk consistently carry two sets of lncRNAs with well-known functions in the inflammatory response and auto-immunity that exhibit opposite loading patterns corresponding to two potential sets of epigenetic exosomal cargo in human breast milk. The specificity and reproducibility of the present study is ensured by the simultaneous co-detection of 'reference IncRNAs' previously identified in the study by Karlsson et al (12) in breast milk-derived exosomes (e.g., GAS5). A novel lncRNA, LINC00657 [non-coding RNA activated at DNA damage (NORAD)] was consistently co-detected with GAS5, which exhibited the highest detection signal among exosomes in all breast milk samples analyzed. Furthermore, the present study represents the first differential expression analysis of breast milk exosomal lncRNAs in breast milk of mothers who gave birth preterm vs. term. The results presented herein indicate that the loading levels of NORAD in breast milk-derived exosomes are suppressed at least 2-fold in mothers who gave birth preterm compared with those who delivered at term, in a statistically significant manner.

\section{Materials and methods}

Study participants. Participants (mothers) between 27-40 years of age without major health issues (e.g., diabetes, toxemia, etc.) and any medication treatment (apart from intake of vitamins, calcium and other supplements) were recruited at the Neonatal Unit of the First Department of Pediatrics of National and Kapodistrian University of Athens at 'Aghia Sophia' Children's Hospital (Athens, Greece) during a 6-month period (January, 2020 to June, 2020). Overall, 20 breast milk samples were collected, 10 from mothers who delivered preterm (gestational age, $<37$ weeks) and 10 from mothers who delivered at term (gestational age, $\geq 37$ weeks). All participants provided written informed consent prior to enrolment, and the study was approved by the Ethics Committee of 'Aghia Sophia' Children's Hospital (protocol code, 24814; date of approval, October 30, 2017).

Breast milk sample collection. Breast milk samples $(20-40 \mathrm{ml})$ were collected using a manual pump at the end of the first month postpartum. Samples were kept at $4^{\circ} \mathrm{C}$ until their transport to the laboratory on the same day. Upon arrival, the 
samples were centrifuged at $1,500 \mathrm{x}$ for $15 \mathrm{~min}$ at $4^{\circ} \mathrm{C}$ and the skim milk fraction was collected through a disposable needle syringe of $5 \mathrm{ml}$. The skim milk sample was centrifuged again at $3,000 \times \mathrm{g}$ for $30 \mathrm{~min}$, at $4^{\circ} \mathrm{C}$ to remove the remaining fat globules and cell debris, and stored in a $1 \mathrm{ml}$ aliquot at $-80^{\circ} \mathrm{C}$ until further analysis.

Isolation of EVs. Breast milk aliquots were thawed on ice and centrifuged at $3,000 \mathrm{xg}$ for $15 \mathrm{~min}$ at $4^{\circ} \mathrm{C}$. The fat layer was discarded using a vacuum and the supernatant was carefully aspirated, mixed with 2 volumes of $1 \mathrm{X}$ PBS and filtered using a $0.8-\mu \mathrm{m}$ membrane unit (EMD Millipore) to remove large aggregates and any residual cell debris. To isolate intact exosomes for western blot analysis, the exoRNeasy Serum/Plasma MaxiKit (Qiagen, Inc.) was used according to the provided protocol. Intact exosomes were extracted from $2 \mathrm{ml}$ of pre-filtered skim milk and were eluted in $140 \mu \mathrm{l}$ of $2 \mathrm{X}$ elution buffer (Qiagen, Inc.).

Western blot analysis. Exosome samples were first lysed using RIPA buffer [cat. no. 89900, Thermo Fisher Scientific, Inc.; $1.0 \%(\mathrm{v} / \mathrm{v}) \mathrm{NP}-40$ or Triton X-100, $0.5 \%$ (w/v) sodium deoxycholate, $0.1 \%(\mathrm{w} / \mathrm{v}) \mathrm{SDS}, 50 \mathrm{mM}$ Tris, $\mathrm{pH} 8.0$ ] and the protein content was quantified using BCA Protein assay (Thermo Fisher Scientific, Inc.). A total of $30 \mu \mathrm{g}$ of each sample were electrophoretically separated on $10 \%(w / v)$ SDS-gel and transferred onto a nitrocellulose membrane $(0.2 \mathrm{~nm})$. The membrane was blocked using $5 \%$ non-fat milk diluted in $1 \mathrm{X}$ TBST (20 mM Tris-base, $150 \mathrm{mM} \mathrm{NaCl,} \mathrm{0,1 \%} \mathrm{w/v} \mathrm{Tween-20),}$ for $1 \mathrm{~h}$ at room temperature. To visualize protein markers, the membrane was incubated with mouse anti-CD63 monoclonal IgG1 antibody (cat. no. sc-5275), mouse anti-CD9 monoclonal IgG1 antibody (cat. no. sc-13118) and mouse anti-cytochrome b5 type b monoclonal IgG1 antibody (cat. no. sc-390876) (all from Santa Cruz Biotechnology, Inc.). All antibodies were diluted 1/500 in 5\% non-fat milk. The samples were incubated for $2 \mathrm{~h}$ at room temperature with anti-mouse secondary IgG Fc antibody HRP-conjugated (cat. no. sc-525409; Santa Cruz Biotechnology, Inc.) diluted $1 / 2,000$ in $5 \%$ non-fat milk. Visualization was performed using Immobilon Forte Western HRP Substrate (cat. no. WBLUF0020; Merck Millipore).

Electron microscopy. An aliquot of $3 \mu \mathrm{l}$ from each sample was added to a grid with a carbon supporting film for $5 \mathrm{~min}$. The excess solution was soaked off using a filter paper, the grid was rinsed by the addition of $5 \mu \mathrm{l}$ distilled water for $10 \mathrm{sec}$, soaked off and stained with $1 \%$ uranyl acetate in water for $10 \mathrm{sec}$ and then air-dried. The samples were examined in a Morgagni 268 transmission electron microscope (FEI Company) at $60 \mathrm{kV}$. Digital images were obtained using a Veleta camera (Olympus Soft Imaging Solutions $\mathrm{GmbH}$ ).

Reverse transcription-quantitative PCR (RT-qPCR). For the extraction of the total RNA content encapsulated in exosomes, the exoRNeasy Serum/Plasma MaxiKit (cat no. 77164; Qiagen, Inc.) was used according to the manufacturer's protocol. RNA was extracted from $4 \mathrm{ml}$ of pre-filtered skim milk and was eluted in $14 \mu \mathrm{l}$ of RNAse-free water. The quality of the isolated total RNA content was assessed using a NanoDrop (ND-1000) spectrophotometer and its concentration was determined using the Qubit RNA assay kit and the Qubit 3.0 fluorometer (Thermo Fisher Scientific, Inc.). The expression of exosomal lncRNAs was determined using a custom 96-well RT ${ }^{2}$ PCR Array (Qiagen, cat. no. CLAH00049). The array was based on the Human Inflammatory Response and Autoimmunity RT $^{2}$ lncRNA PCR array (cat. no. LAHS-004Z; Qiagen, Inc.) consisting of 84 lncRNAs ( $\mathrm{RT}_{2}$ lncRNA PreAMP primer mix; Qiagen $\mathrm{GmbH}$; contains pre-dispensed, laboratory verified, gene-specific primer pairs for 84 genes) involved in autoimmune and inflammatory immune responses, as well as three lncRNAs that were reported previously by Karlsson et al (12) to be consistently detected in human breast milk-derived EVs (SRA1, CRNDE and DANCR). This panel also included a set of controls to evaluate genomic DNA contamination, as well as PCR and reverse transcription performance. cDNA synthesis was performed using the RT2 First Strand kit (ID:330401, custom cat. no. CLAH00049-LAHS-004Z; Qiagen, Inc.) according to the manufacturer's protocol that includes a DNA elimination step prior the reverse transcription. For the cDNA synthesis, $1 \mu \mathrm{g}$ of RNA was diluted in RNase-free $\mathrm{H}_{2} \mathrm{O}$ to a final volume of $8 \mu \mathrm{l}$ (without a pre-amplification step) was used as an input. The synthesized cDNA was then mixed with RT2 SYBR-Green MasterMix and RNase-free water to yield the PCR component, which was distributed in $25 \mu \mathrm{l}$ aliquots to each well of the lncRNA PCR plate. qPCR was performed on a Light cycler 480 II PCR machine (96-well; Roche Diagnostics) and the PCR cycling conditions consisted of an initial step of $10 \mathrm{~min}$ at $95^{\circ} \mathrm{C}$ for HotStart Activation, followed by 40 cycles of $15 \mathrm{sec}$ at $95^{\circ} \mathrm{C}$ and $1 \mathrm{~min}$ at $60^{\circ} \mathrm{C}$. A $\mathrm{Cq}$ value $<40$ was considered as the limit for the detection of expression. Normalized expression levels were calculated using inverse Min-max feature scaling, $1-\left[\mathrm{NorCq}_{\text {target }}\right.$ lncRNA-MIN(NorCq)]/[MAX(NorCq)-MIN(NorCq)], where NorCq $=\mathrm{Cq}_{\text {target }} \operatorname{lncRNA} / \mathrm{Cq}_{\text {Reference }}$, while $\mathrm{Cq}_{\text {Reference }}=\left(\mathrm{Cq}_{\mathrm{RPLP0}}+\right.$ $\left.\mathrm{Cq}_{\text {Aств }}\right) / 2$. To investigate the differences between the expression of lncRNAs in the breast milk of mothers who delivered infants at term and those who delivered preterm, raw data were analyzed and the relative expression level of target lncRNAs was determined using the $2^{-\Delta \mathrm{Cq}}$ method, where $\Delta \mathrm{Cq}=\mathrm{Cq}_{\text {target }}$ IncRNA-Cq $q_{\text {Reference }}$ (24). Ribosomal protein lateral stalk subunit P0 (RPLPO) and actin beta $(A C T B)$ were selected as reference genes to minimize the standard deviation (SD) of expression. lncRNAs were considered differentially expressed if the fold change was $>2$ or $<0.5$.

Statistical analysis. P-values were calculated between two groups (10 preterm and 10 full-term breastmilk samples) using the t-test in programming language $\mathrm{R}$ (package 'stats', version 4.2.0) and the statistical significance threshold was set to 0.05 . The correlation of expression was estimated using Pearson's coefficient which reflects the linear correlation between two variables accounting for differences in their mean and SD.

\section{Results}

Isolated exosomes from human breastmilk samples from mothers who gave birth at term or preterm were labeled as positive for two common exosomal tetraspanins, CD9 and CD63, using western blot analysis (Fig. 1A). To examine exosome 
A

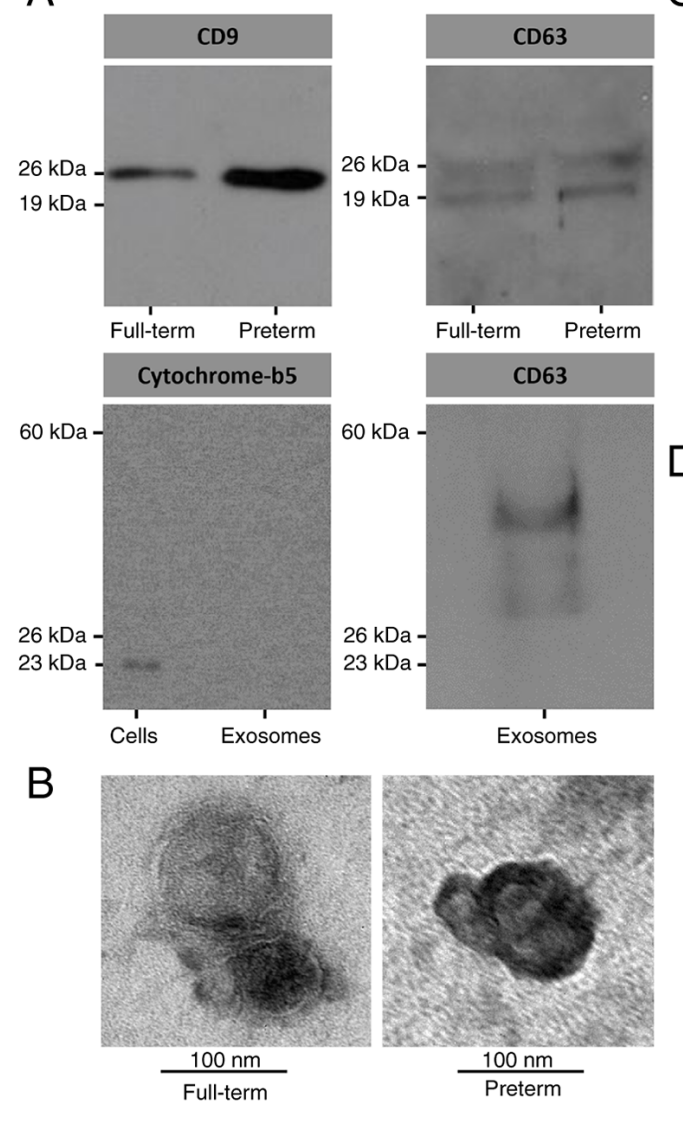

C

\begin{tabular}{|c|c|c|c|c|c|c|c|c|c|c|}
\hline Sample & Pregnancy & $\begin{array}{l}\text { Gestational } \\
\text { age (weeks) }\end{array}$ & Nationality & Age & $\begin{array}{l}\text { Weight } \\
(\mathrm{kg})\end{array}$ & $\begin{array}{l}\text { Height } \\
(\mathrm{cm})\end{array}$ & $\begin{array}{l}\text { Waist circumference } \\
(\mathrm{cm})\end{array}$ & BMI & $\begin{array}{c}\text { Child weight } \\
(\mathrm{kg})\end{array}$ & $\begin{array}{l}\text { Child height } \\
\text { (cm) }\end{array}$ \\
\hline Sample 1 & Preterm & 27 & Russia & 37 & 82 & 174 & 97 & 26.9 & 1.200 & 37.0 \\
\hline Sample 2 & Preterm & 29 & Albania & 24 & 76 & 165 & 94 & 28.0 & 1.130 & 37.0 \\
\hline Sample 3 & Preterm & 29 & Romania & 17 & 46 & 146 & 78 & 21.5 & 1.590 & 44.0 \\
\hline Sample 4 & Preterm & 29 & Greek & 35 & 58 & 165 & 71 & 21.6 & 1.170 & 43.0 \\
\hline ample 5 & Preterm & 31 & Greek & 42 & 54 & 166 & 88 & 19.6 & 1.800 & 43.0 \\
\hline Sample 6 & Preterm & 32 & Greek & 37 & 65 & 170 & 87 & 22.5 & 1.465 & 41.0 \\
\hline Sample 7 & Preterm & 32 & Greek & 28 & 90 & 176 & 88 & 29.1 & 1.370 & 41.0 \\
\hline Sample 8 & Preterm & 33 & Greek & 36 & 60 & 164 & 87 & 22.3 & 1.680 & 52.5 \\
\hline Sample 9 & Preterm & 33 & Greek & 30 & 69 & $\begin{array}{l}160 \\
160\end{array}$ & 91 & $\begin{array}{l}27.0 \\
27.0\end{array}$ & 1.780 & 43.5 \\
\hline ample 10 & 0 Preterm & 36 & Greek & 43 & 63 & 163 & 91 & 23.7 & 2.670 & 48.0 \\
\hline Sample 11 & 1 Full-term & 38 & Greek & 38 & 78 & 16 & 102 & 29.4 & 4.290 & 55.0 \\
\hline Sample 12 & 2 Full-term & 38 & Greek & 38 & 55 & 163 & 73 & 20.7 & 3.100 & 50.0 \\
\hline Sample 13 & 3 Full-term & 38 & Greek & 29 & 75 & 163 & 100 & 28.2 & 3.800 & 52.0 \\
\hline Sample 14 & 4 Full-term & 38 & Greek & 34 & 50 & 165 & 78 & 18.4 & 3.300 & 51.0 \\
\hline Sample 15 & 5 Full-term & 39 & Greek & 36 & 75 & 168 & 93 & 26.6 & 2.500 & 48.0 \\
\hline Sample 16 & 6 Full-term & 39 & Albania & 37 & 61 & 16 & 88 & 32.3 & 3.300 & 51.0 \\
\hline Sample 17 & 7 Full-term & 39 & Greek & 37 & 60 & 16 & 89 & 22.9 & 2.900 & 50.0 \\
\hline Sample 18 & 8 Full-term & 39 & Greek & 28 & 49 & 15 & 72 & $\begin{array}{l}19.4 \\
19.5\end{array}$ & 3.260 & 52.0 \\
\hline Sample 19 & 9 Full-term & 39 & Greek & 33 & 76 & 165 & 100 & 27.9 & 3.520 & 53.0 \\
\hline Sample 20 & Full-term & 40 & Greek & 33 & 90 & 163 & 103 & 33.9 & 2.900 & 54.0 \\
\hline
\end{tabular}

$D_{s}$

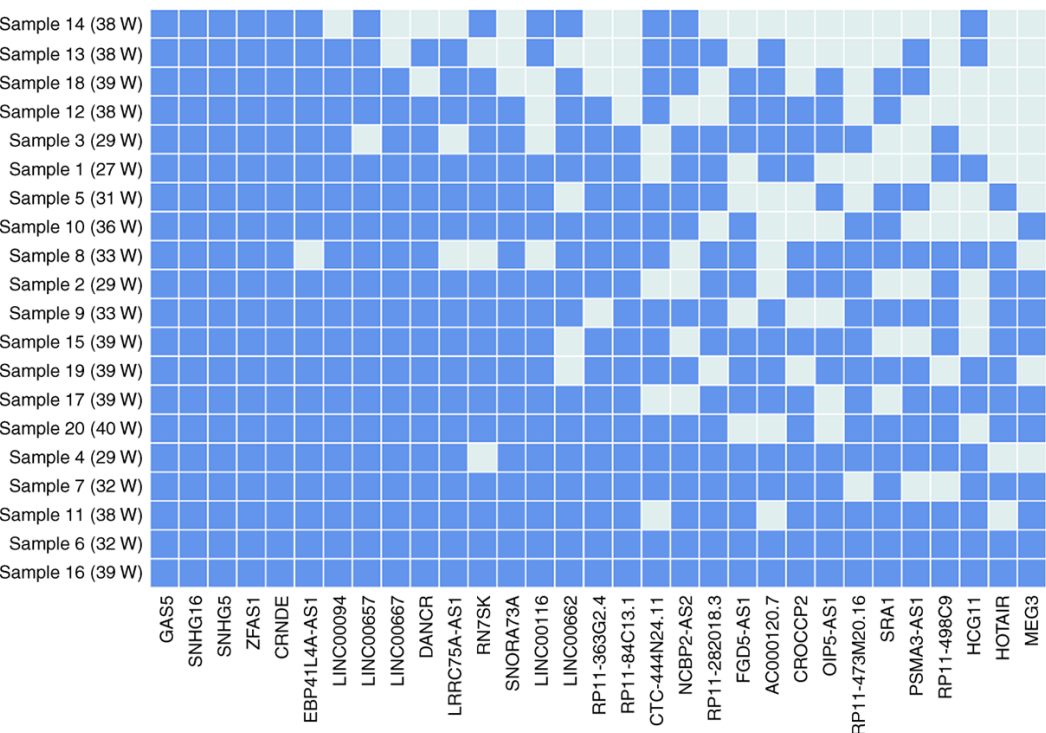

Figure 1. Exosome characterization. (A) Isolated exosomes from human breastmilk samples from mothers who delivered at term or preterm were labeled as positive for two common exosomal tetraspanins, $\mathrm{CD} 9$ and CD63, and negative for a protein of outer mitochondrial membrane, cytochrome-b5 type b, using western blot analysis. (B) Size and morphology of exosomes in breastmilk from mothers who delivered at term and preterm. (C) Demographic and anthropometric characteristics of the mothers and infants studied. (D) Panel showing the expression patterns of all lncRNAs detected in $\geq 50 \%$ of the samples. Cells with blue color represent samples where lncRNAs were detected using reverse transcription-quantitative PCR with $<40$ Cq values, while cells with white color represent samples with undetected $\operatorname{lncRNAs}(>40 \mathrm{Cq}$ value). IncRNAs were sorted according to their Cq values, beginning with lncRNAs that were most frequently detected. IncRNAs located on the left of the red dashed line were detected at least in $85 \%$ of all samples. IncRNA, long non-coding RNA.

purity, western blot analysis was performed for cytochrome-b5 type b, a common cell marker of the outer mitochondrial membrane. The results revealed that the cells were positive for cytochrome-b5 type $\mathrm{b}$, while the exosomes exhibited no signal, thus suggesting no evidence of cell contamination. To further characterize the composition of the exosomes in the present study, western blot analysis was performed using an antibody against CD63 as an additional positive tetraspanin marker for exosomes. Again, the exosome lysates were positive for CD63. Of note, the CD9 protein levels in the exosomes derived from the breastmilk of mothers who delivered preterm were markedly higher compared with those in the breastmilk from mothers who delivered at term, even though equal protein quantities of whole exosome lysates were loaded to the gel. Subsequently, exosomal RNA extraction from all samples was performed. To address the issue regarding the size and morphology of exosomes derived from breastmilk from mothers who delivered at term and preterm, electron microscopy was performed. The results indicated that the exosomes isolated from breastmilk exhibited the classical 'doughnut' morphology due to the surrounding lipid bilayer and their size was between 50-100 nm (Fig. 1B). The demographic and anthropometric characteristics of the mothers, as well their children, are presented in Fig. 1C.

In order to study the IncRNA cargo of human breastmilk-derived exosomes, 20 RNA samples were isolated from exosomes of 10 breastmilk samples obtained from mothers who delivered at term ( $\geq 37$ weeks of gestation) and an equal number of samples from mothers with preterm birth ( $<37$ weeks of gestation). Subsequently, RNA samples were analyzed using RT-qPCR. The analysis was performed using a customized panel of targets against lncRNAs with well documented roles in the inflammatory response and auto-immunity, as well as selected lncRNAs that were previously reported to be significantly detected in human breastmilk-derived exosomes (12). Following RT-qPCR, 76 out of 88 lncRNAs were detected in at least one sample. Furthermore, 31 lncRNAs were detected as an exosomal load in $\geq 50 \%$ of the samples (Fig. 1D). Importantly, 13 lncRNAs were consistently detected in the breastmilk-derived exosomes in $\geq 85 \%$ of the samples (Table I). The biological functions of these lncRNAs are presented in detail at Table SI.

In the heatmap analysis of the normalized expression levels of selected lncRNAs detected in $\geq 50 \%$ of the samples (Fig. S1), 
Table I. IncRNA expression levels detected in $>85 \%$ of the breastmilk samples from mothers who delivered preterm and or at full-term.

\begin{tabular}{lccccc}
\hline lncRNA ID & $\begin{array}{c}\text { Mean } \Delta \text { cq preterm } \\
( \pm \text { SD })\end{array}$ & $\begin{array}{c}\text { Mean } \Delta \text { cq full-term } \\
( \pm \text { SD })\end{array}$ & Sample no. & $\log _{2}$ fold change & P-value \\
\hline LINC00657 & $3.78( \pm 0.61)$ & $2.79( \pm 1.44)$ & $19 / 20$ & -0.996 & 0.034 \\
LRRC75A-AS1 & $2.90( \pm 0.65)$ & $3.62( \pm 0.95)$ & $17 / 20$ & 0.713 & 0.045 \\
CRNDE & $3.35( \pm 1.00)$ & $2.20( \pm 1.83)$ & $20 / 20$ & -1.144 & 0.052 \\
SNHG16 & $4.55( \pm 0.60)$ & $4.13( \pm 0.78)$ & $20 / 20$ & -0.413 & 0.102 \\
ZFAS1 & $1.78( \pm 0.76)$ & $1.24( \pm 1.09)$ & $20 / 20$ & -0.541 & 0.108 \\
SNHG5 & $1.49( \pm 0.57)$ & $0.86( \pm 1.79)$ & $20 / 20$ & -0.629 & 0.156 \\
SNORA73A & $6.73( \pm 1.88)$ & $6.12( \pm 1.77)$ & $17 / 20$ & -0.617 & 0.251 \\
DANCR & $5.51( \pm 1.17)$ & $5.77( \pm 0.85)$ & $18 / 20$ & 0.256 & 0.299 \\
LINC00667 & $5.84( \pm 1.55)$ & $6.06( \pm 1.30)$ & $18 / 20$ & 0.221 & 0.373 \\
GAS5 & $1.64( \pm 1.92)$ & $1.39( \pm 1.55)$ & $20 / 20$ & -0.251 & 0.376 \\
EBP41L4A-AS1 & $5.61( \pm 0.99)$ & $5.44( \pm 1.63)$ & $19 / 20$ & -0.169 & 0.393 \\
LINC00094 & $6.17( \pm 1.42)$ & $6.25( \pm 0.94)$ & $19 / 20$ & 0.080 & 0.443 \\
RN7SK & $4.89( \pm 1.24)$ & $4.84( \pm 1.12)$ & $17 / 20$ & -0.047 & 0.468 \\
\hline
\end{tabular}

lncRNA, long non-coding RNA.

GAS5 lncRNA exhibited the highest degree of loading levels in the breastmilk exosomes among all the lncRNAs analyzed. Following the correlation heatmap analysis of the normalized expression data, two clusters of lncRNAs were identified as differentially co-detected across samples (Fig. 2A). The first cluster (blue color) included 10 lncRNAs [CTC-444N24.11, LINC00657, NCBP2-AS2, CRNDE, HCG11, small nucleolar RNA host gene (SNHG16), GAS5, EBP41L4A-AS1, SNHG5 and $Z F A S 1]$ exhibiting a positive intergroup expression correlation and a negative correlation with the majority of the remaining lncRNAs. In the aforementioned group, 7 out of the 10 lncRNAs co-loaded in the breastmilk exosomes were detected consistently across the samples $(\geq 85 \%)$. The second cluster (orange color) included a much larger subset of lncRNAs (HOTAIR, MEG3, RP11-282018.3, DANCR, RP11-363G2.4, SNORA73A, RP11-473M20.16, RP11-498C9, RP11-84C13.1, CROCCP2, LINC00094, LINC00667, LRRC75A-AS1 and RN7SK); six of these (DANCR, LINC00094, LINC00667, SNORA73A, LRRC75A-AS1 and RN7SK) were consistently detected in $\geq 85 \%$ of the samples.

This study then aimed to cross-validate these two novel opposing correlation patterns by analyzing only the 13 lncRNAs with the highest frequency across samples ( $\geq 85 \%$ of samples) (Fig. 2B). Correlation analysis again revealed the existence of two distinct loading lncRNA patterns in the breastmilk-derived exosomes with a strong inverted correlation. More precisely, six IncRNAs (GAS5, SNHG5, ZFAS1, LINC00657, SNHG16 and $C R N D E$ ) formed a prominent group of abundantly co-detected lncRNAs that exhibited strong negative correlation with a subset of four different lncRNAs (DANCR, LINC00094, LINC00667 and $S N O R A 73 A$ ) that formed the core of the second cluster of abundantly co-detected lncRNAs (Fig. 2C). The identification of two differentially co-detected sub-sets of lncRNA cargo in breastmilk-derived exosomes may indicate their co-regulation and common loading in the same breastmilk-derived exosomes, in a distinct and mutual exclusive manner across samples.
A differential expression analysis of the lncRNAs loaded in the exosomes of breastmilk samples from mothers who delivered preterm compared to those who delivered at term was conducted. The results were obtained as a volcano plot of the $\log 2$ fold change of lncRNAs (Fig. 3A) and corresponding values for statistical significance (P-value; $-\log _{10} \mathrm{P}$ ) (up- and downregulated lncRNAs are presented in Tables SII and SIII, respectively). In total, nine $\operatorname{lncRNAs}$ exhibited at least $\geq 1 \log _{2}$ fold change (one upregulated and eight down-regulated lncRNAs), which indicated that their levels were at least 2-fold up- or downregulated in the breastmilk of mothers who delivered prematurely compared to those who gave birth at term. In addition, one lncRNA (LRRC37BP1) was upregulated at least 2-fold, although with no statistically significant difference (t-test $\mathrm{P}$-value $=0.333, \log _{2}$ fold change=1.076). By contrast, out of the eight downregulated $\operatorname{lncRNAs}$ with $\geq 1 \log _{2}$ fold change, two exhibited statistically significant differences (t-test $\mathrm{P}$-value $\leq 0.05$ ). Thus, the levels of exosomal lncRNAs appeared to be mostly downregulated in the breastmilk of mothers who delivered preterm. LINC00657 (NORAD) $(\mathrm{P}$-value $=0.034$, $\log _{2}$ fold change $=-1.00$ ) was the only $\operatorname{lncRNA}$ in the current panel that was consistently detected in $\geq 85 \%$ of the samples, clustering well together with previously reported lncRNAs in breastmilk-derived exosomes and exhibiting a statistically significant difference ( $\geq 2$-fold downregulation).

Likewise, IncRNA $C R N D E$ was downregulated in the exosomes of breastmilk-from mothers who delivered preterm, although with a fractionally acceptable P-value of $0.052\left(\log _{2}\right.$ fold change $=-1,14$ ), while $C T C-444 N 24.11$ was downregulated with a $\log _{2}$ fold change of -1.521 and a P-value of 0.048 . However, CTC-444N24.11 was detected only in 50\% of the samples. By contrast, LRRC75A-AS1 was upregulated with a statistically significant difference $(\mathrm{P}$-value $=0.045)$, albeit it exhibited a $<2$-fold chance $\left(\log _{2}\right.$ fold change $\left.=0.713\right)$. More specifically, the expression levels of the four lncRNAs which exhibited statistically significant differences (LINC00657, 

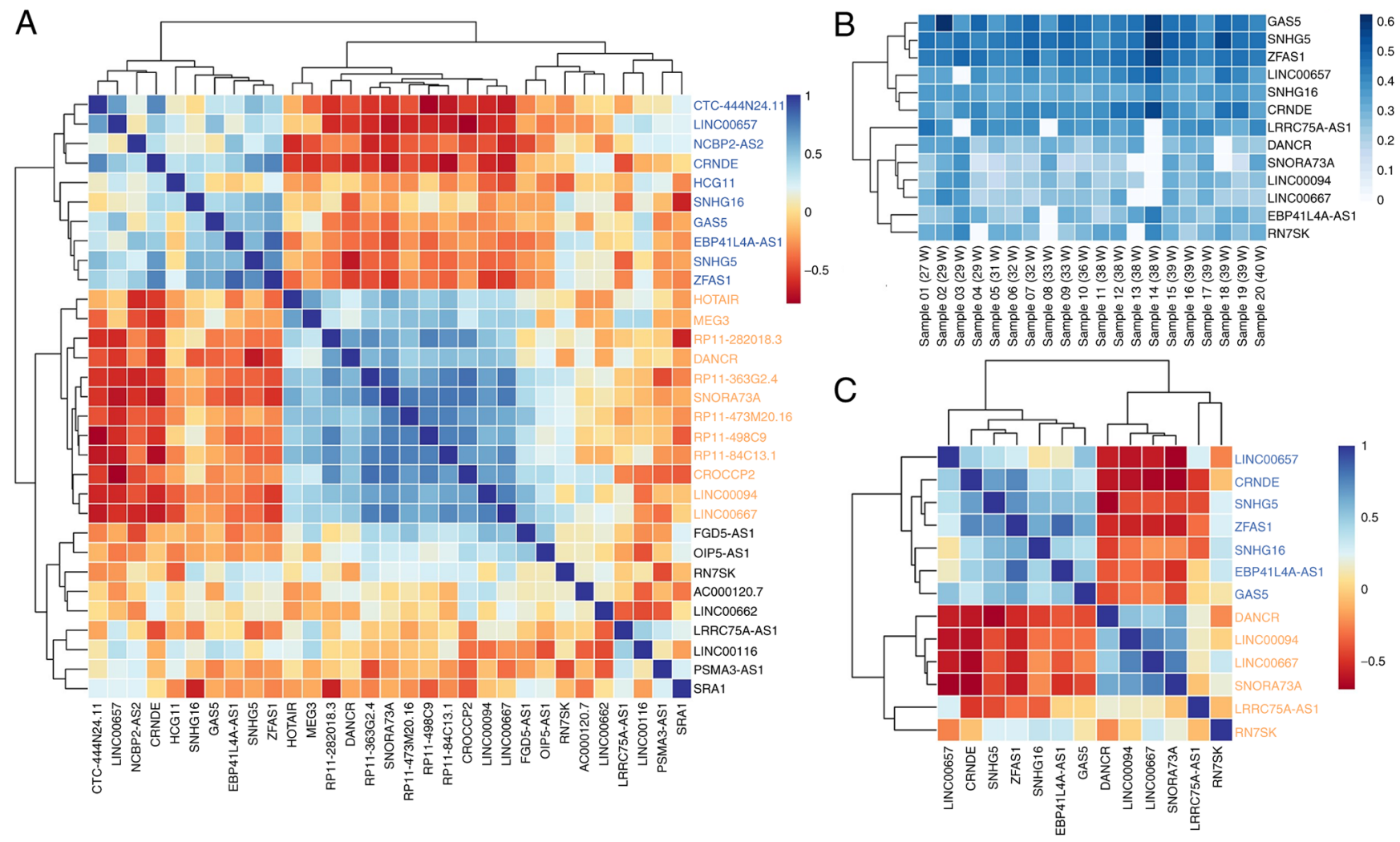

Figure 2. Reverse transcription-quantitative PCR-based expression of lncRNAs. (A) Correlation heatmap of normalized expression data for lncRNAs detected in $\geq 50 \%$ of the samples. Pearson's correlation analysis was used among the 31 lncRNAs to reveal the co-expression patterns of lncRNAs, while hierarchical cluster illustrates grouping patterns. Positive correlations are marked in blue and negative ones in red. (B) Heatmap of normalized expression levels of lncRNAs detected in $\geq 85 \%$ of samples. lncRNA expression levels are represented in blue (higher expression). (C) Correlation heatmap of normalized expression data for lncRNAs detected in $\geq 85 \%$ of the samples. lncRNA, long non-coding RNA.

$C T C-444 N 24.11, C R N D E$ and $L R R C 75 A-A S 1)$ across all samples, in both the term and preterm group, are presented in bar plots in Fig. 3B. The lncRNAs LINC00657 (NORAD) and $C R N D E$ were both found to be: i) Detected in $\geq 85 \%$ of the samples; ii) downregulated $\geq 2$-fold in exosomes in breastmilk from mothers who delivered preterm compared to those who delivered at term; and iii) positively correlated with a signature of abundantly detected IncRNAs composed of previously reported, as well as newly identified lncRNAs. Based on the aforementioned results, it was reported that lncRNA NORAD was downregulated in a statistically significant manner in exosomes in breastmilk from mothers who gave birth prematurely while it was, consistently and specifically, co-detected in breastmilk-derived exosomes. The findings described above suggest that lncRNA NORAD represents a specific loading signature of lncRNA cargo in breastmilk-derived exosomes (lncR NAs positively and negatively correlated with NORAD levels in breastmilk exosomes are presented in Table SIV).

\section{Discussion}

Breastmilk is abundant in exosomes/EVs that have a higher propensity for therapeutic effects, while also having the potential to be easily administered clinically (25). In addition, milk-derived exosomes exhibit an increased survivability following simulated gastric/pancreatic digestion (26). There is emerging evidence to indicate that exosome-encapsulated RNAs can regulate target cell pathways related to cellular growth, division, differentiation, stress response, survival, apoptosis, metabolism and immunity (14). Recent discoveries have revealed the presence of IncRNAs in breastmilk-derived exosomes, with well-documented roles in development (12). In addition, it has been demonstrated that the protein levels of CD9, CD63 and CD81 tetraspanins are consistently higher in exosomes in breastmilk from mothers who deliver preterm compared to those in breastmilk from mothers who delivered at term. The increased CD9 expression observed in breastmilk from mothers who deliver preterm may constitute an additional signature of preterm birth that reflects the difference in $\mathrm{EV}$ constitution and it remains to be determined if it plays a functional role in mother-child communications (27). In the present study, 13 key lncRNAs were consistently and robustly detected in exosomes across $85 \%$ of all breastmilk samples, reaffirming the findings of the study by Karlsson et al (12), but also significantly expanding the list to include lncRNAs with well-documented roles in inflammation, auto-immunity, metabolism, cell cycle control and cell differentiation, and thus in the development of neonates. Among these, ten lncRNAs (CTC-444N24.11,LINC00657, NCBP2-AS2, CRNDE, HCG11, SNHG16, GAS5, EBP41L4A-AS1, SNHG5 and ZFAS1) were highly expressed concurrently in all samples, and importantly, they belong to the same gene cluster, suggesting their highly positive correlation. The correlation of expression regarding lncRNAs detected in $\geq 50 \%$ of the samples revealed two distinct clusters that negatively correlated with each other. Namely, the increased expression levels of the first cluster signify decreased 
A
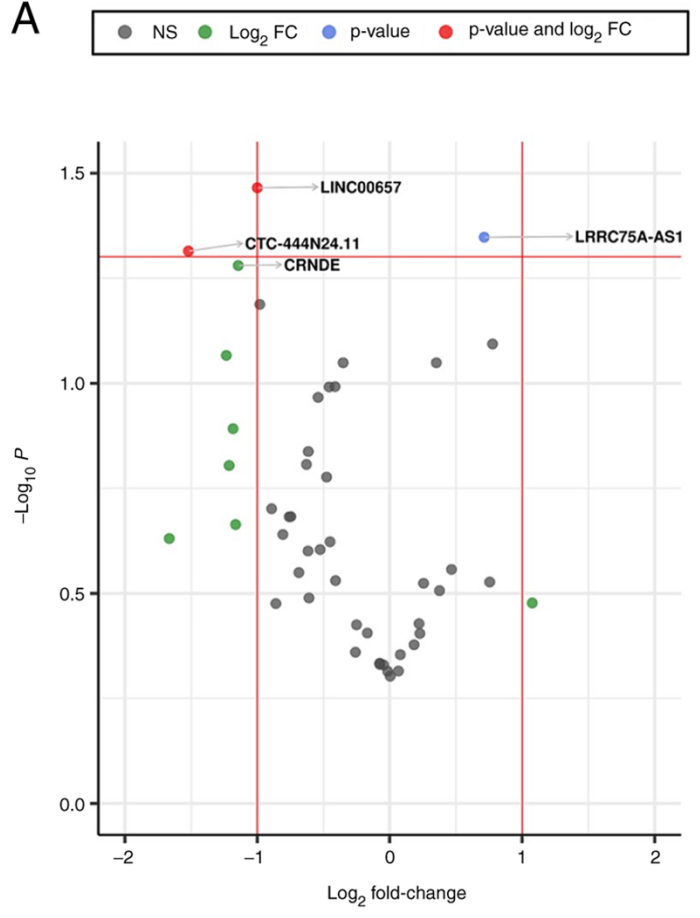

B
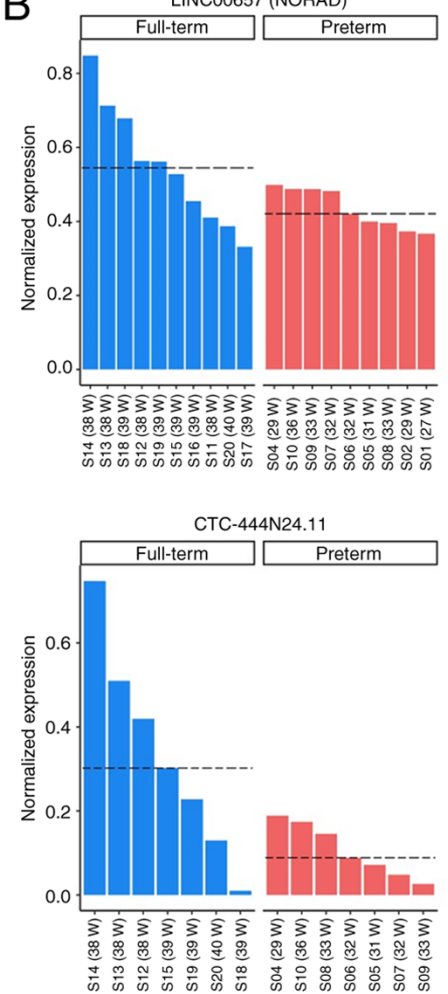
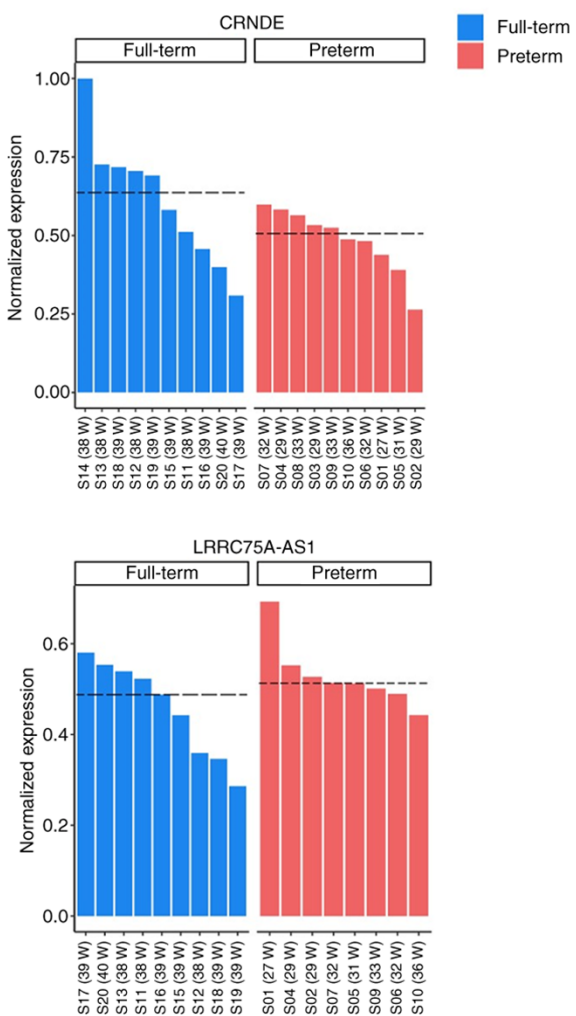

Figure 3. Differential lncRNA expression analysis in breastmilk samples between mothers who delivered preterm and at full-term. (A) Volcano plot showing the degree of differential expression. $\log _{2}$ (fold change) for expression is plotted on the $\mathrm{x}$-axis, whereas the negative $\log _{10}(\mathrm{P}$-value) is displayed on the $\mathrm{y}$-axis. Vertical red lines delineate a 2 -fold change ( $\log _{2}$ fold change, $\geq 1$ or $\left.\leq-1\right)$ and each point represents one lncRNA. NS (grey), non-significant fold change ( $\log _{2}$ fold change, $\leq 1$ or $\geq-1$ ); $\log _{2} \mathrm{FC}$ (green), significant fold change but non-significant P-value; P-value (blue), non-significant fold change, but significant P-value; $\mathrm{P}$-value and $\log _{2} \mathrm{FC}$ (red), both fold change and P-value were significant). P-value $<0.05$ (horizontal red line) was considered to indicate a statistically significant difference. (B) Bar graph representations of per sample lncRNA normalized expression for full-term (blue) and preterm (red) groups. The top 4 differentially? expressed lncRNAs according to P-value are depicted in this plot. The horizontal dashed line represents the median value of expression for each group. lncRNA, long non-coding RNA.

levels of the second one. The lncRNAs of each cluster exhibited a positive correlation among them, reflecting a potential regulatory mechanism of lncRNA loading to breastmilk exosomes. These results are likely to be related to the function of each IncRNA, as it can be hypothesized that lncRNAs of the same cluster employ similar function at recipient cells, in this case, contributing to the development of infants.

GAS5 was the most highly expressed lncRNA in breastmilk-derived exosomes. GAS5 is known to be activated in response to growth arrest and cellular starvation due to lack of nutrients or growth factors. It functions as a riborepressor, regulating glucocorticoid receptor, by binding to its DNA binding domain through glucocorticoid response element (28). Importantly, GAS5 is involved in immunity and plays a vital role in moderating the normal growth arrest of T-cells and non-transformed lymphocytes, as well as macrophage polarization $(29,30)$. Consequently, GAS5 appears to play an essential role in programming the neonatal immune system. Furthermore, the present study provides new insight on the differential content of lncRNAs in EVs from breastmilk obtained from mothers who delivered at term compared to those who delivered preterm. In total, eight lncRNAs were downregulated, having at least $\geq 1 \log _{2}$ fold change, while only one IncRNA was found to be upregulated in the breastmilk of mothers who delivered prematurely. LRRC75A-AS1 was only marginally upregulated, but exhibited a statistically significant difference $(\mathrm{P}$-value=0.045). LRRC75A-AS1, also known as SNHG29, has previously been shown to be associated with spontaneous preterm birth. According to the study by Jiang et al (31), oxidative stress regulates SNHG29, which in turn accelerates cellular senescence and triggers pro-inflammatory cytokine release from senescent cells. SNHG29 was found to be upregulated in the placentas of women who underwent preterm labor (31). Thus, the results of the present study further strengthen the association between SNHG29 and preterm birth.

Among the lncRNAs found to be downregulated in the exosomes in breastmilk from mothers who delivered prematurely, three (LINC00657, CTC-444N24.11 and CRNDE) were downregulated in a statistically significant manner; notably, they belong to the same cluster, thus indicating that a common suppression mechanism(s) may be responsible for their differential regulation in exosomes in breastmilk from mothers who delivered preterm. IncRNA CTC-444N24.11 was found to be significantly downregulated with $a \geq 2$-fold change $(\mathrm{P}$-value $=0.048)$ in the breastmilk of mothers who delivered preterm birth compared to those who delivered at term. To date, the role of CTC-444N24.11 remains unknown, while by reviewing the literature no data were found exclusively for $C T C-444 N 24.11$. In a recent bioinformatics analysis of miRNA expression in retinopathy of prematurity (ROP), CTC-444N24.11 was indicated as a target of miRNA-128-3p, 
which was downregulated in premature infants with ROP (32). $C R N D E$ was also found to display a $\geq 2$-fold differential expression and can be referred to as a marginally significant (P-value $=0.052)$ differentially expressed lncRNA. CRNDE is a central regulator of glucose and lipid metabolism, while it is regulated by insulin and insulin growth factors (33). It regulates cyclin D1 and consequently, normal cell division (34). Its role in normal cellular developmental and pluripotency (35) possibly indicates the importance of its presence in breastmilk in normal infant's development.

Only LINC00657 was found to be ubiquitously detected in breastmilk-derived exosomes and was also significantly downregulated ( $\geq 2$ fold change) in breastmilk form mothers who delivered preterm. LINC00657 is a long intergenic ncRNA named NORAD (HGNC ID), for non-coding RNA activated by DNA damage. NORAD is a cytoplasmic IncRNA, and is one of the most abundant and highly conservative lncRNAs among mammalian cells and species, respectively (36). Of note, due to tandem sequence duplication that occurred during evolutional processes, NORAD consists of 12 repeated units, which facilitate its function (37). NORAD is referred to as the guardian of genome, while mounting evidence suggests its crucial role in maintaining genome stability. NORAD binds Pumilio RNA-binding proteins (PUM 1 and PUM 2), functioning as a decoy, and inhibits them from repressing their mRNA targets, which are key regulators of DNA repair and replication, mitosis and mitochondrial homeostasis $(38,39)$. In addition, NORAD functions as scaffold, interacting with RNA binding motif protein X-linked (RBMX), a component of DNA damage response, and mediates the assembly of a ribonucleoprotein complex [NORAD-activated ribonucleoprotein complex 1 (NARC1)], which contains the known suppressors of genomic instability topoisomerase I (TOP1), Aly/REF export factor (ALYREF) and the pre-mRNA processing factor 19 (PRPF19)-cell division cycle 5 like (CDC5L) complex (40). Another study revealed the role of NORAD in regulating nuclear translocation and signal transduction. More precisely, NORAD interacts with importin- $\beta 1$ and therefore, regulates Smad translocation into the nucleus and TGF- $\beta$ signaling (41).

The existence of lncRNA NORAD in exosomes may serve as an important signaling molecule in the adaptive responses of the infant after birth to hypoxic conditions. NORAD lncRNA has been found to be upregulated during hypoxic conditions (42) and as such, NORAD can participate in the adaptive mechanism of the newborns during the perinatal period; i.e., to combat the high levels of oxygen to which they are exposed after birth. Failure to adapt to higher-than-normal levels of oxygen leads to the induction of oxidative stress and the generation of reactive oxygen species (43). Critically, previous research point towards a higher level of oxidative stress in preterm newborns than those born full-term (43) due to immature and not fully developed respiratory, digestive, immune and antioxidant defense systems. Thus, the downregulation of lncRNA NORAD in the breastmilk of mothers who delivered prematurely in the present study, is in line with the findings of previous research reporting elevated levels of oxidative stress biomarkers (8-OHdG, hydroperoxide, malondialdehyde, etc.) and a lower activity of antioxidant enzymes (superoxide dismutase, glutathione peroxidase, etc.) in preterm babies. Importantly, the formula feeding of preterm infants has been associated with higher levels of oxidative stress than breastmilk feeding and, as such, the existence of NORAD in exosomes together with the known nutritional antioxidant content of breast milk may help the newborn to adapt to the higher levels of oxidative stress (43). Thus, NORAD may be an important therapeutic target with notable consequences for premature infants who exhibit higher levels of OS (43).

It is important to take into consideration the limitations of the present study. The main limitation is the small number of samples used. However, these are preliminary results. Larger numbers of breastmilk samples are required to increase the statistical validity of the results presented herein. Another limitation in determining lncRNA expression levels involves the lack of an accurate exosomal reference gene. Nevertheless, two genes Actin and RPLP0 were used as reference genes in order to diminish the possibility of false results (i.e., based on randomness). The currents findings provide some insight into the upcoming field of exosomal research, breaking new ground and providing promising evidence in understanding the role of epigenetic mechanisms, providing a good starting point for discussion and future research. Integrated high-throughput technologies can complement the results of the present study by elucidating the type of physiological complexity with higher precision. Translating these technologies into clinical practice in the form of a non-invasive tests will help realize in the near future, the personalization of medicine. Even though the miRNA transcriptome of exosomes in human breastmilk has been uncovered $(13,22,23)$ and the miRNA expression dynamics between full-term and preterm breastmilk's lipid fractions have been characterized $(26,44)$, the exosome-enriched lncRNA populations in the two groups mentioned above remain unknown.

Not with standing these limitations, the present study validates and expands previous findings that specific IncRNAs are loaded in EVs derived from breastmilk, possibly by a common regulatory mechanism, and are thus, detected together in a highly correlated manner. NORAD appears to belong to the same loading/regulatory mechanism; however, it represents the first lncRNA to date, whose levels are differentially regulated and significantly suppressed in human breastmilk from mothers who delivered prematurely. The absence of NORAD among others, such as impaired mitotic division, mitochondrial dysfunction, premature cell aging and neuronal dysfunction, contributes to replication-related stress and DNA damage. If further validated as true, a significant downregulation of NORAD exosomal cargo in the breastmilk of mothers who deliver preterm may serve as an exosomal biomarker for replication-related stress and may be associated with inflammatory and immune responses in preterm infants. Finally, NORAD may be directly used as a therapeutic agent. It could be reverse-engineered to be enriched in exosomes from human mammary stem cells isolated from mother's own breastmilk and added even on a given milk formula.

In conclusion, breastmilk-derived exosomes are important mediators of communication between mother and child, while IncRNAs may play a crucial role in neonatal growth and development, in a short- and long-term manner. Collectively, the data presented herein reveal the consistent co-detection of IncRNAs that are implicated in several key processes, such as immune system development, metabolism and cell cycle 
control, as well as providing a potential mechanism regarding their load in breastmilk-derived EVs in a highly correlated manner. To the best of our knowledge, this is the first study to analyze the differential expression of lncRNAs in breastmilk between term and preterm mothers. NORAD is a lncRNA involved in the DNA damage response and repair pathway, referred to as the guardian of the human genome and among others, found to be significantly downregulated at least 2-fold in the breastmilk of preterm mothers. Broadly translated, this result indicates that the absence of NORAD may serve as an exosomal biomarker for replication-related stress in preterm infants. Notably, NORAD and other lncRNAs could be produced by reverse-engineering and added to breastmilk or other milk formulas and may function therapeutically in preterm infants. Future research is required to analyze lncRNA expression and function in breastmilk from mothers who deliver at term compared to those who deliver preterm on a larger scale. This will possibly shed more light on lncRNA involvement and impact on children's development and health.

\section{Acknowledgements}

Not applicable.

\section{Funding}

The present study was funded by the project 'MilkSafe: A novel pipeline to enrich formula milk using omics technologies (MIS 5074548)' which is implemented under the Action 'Reinforcement of the Research and Innovation Infrastructure', funded by the Operational Programme 'Competitiveness, Entrepreneurship and Innovation' (NSRF 2014-2020) and co-financed by Greece and the European Union (European Regional Development Fund).

\section{Availability of data and materials}

The RT-qPCR data [Cq values (raw data)] corresponding to exosomes from breastmilk samples that support the findings of this study are available in figshare public databases (https://doi. org/10.6084/m9.figshare.16635592).

\section{Authors' contributions}

TS, AG, AS and GPC were involved in the conceptualization of the study. AG, NM, DV, MP, TS and GPC were involved in the study methodology. AG, NM, EK, AK, GL, GB, MT and AT were involved in formal analysis (data acquisition, data analysis and in the experiments). AG, TS, AS, NM, EK, DV and GPC were involved in the writing and preparation of the original draft. AG, MT, AT, GB, DV and GPC were involved in the writing, reviewing and editing of the manuscript. GPC and $A G$ were responsible for the acquisition of funding and confirm the authenticity of all the raw data. All authors have read and agreed to the published version of the manuscript.

\section{Ethics approval and consent to participate}

The study was conducted according to the guidelines of the Declaration of Helsinki and approved by the Institutional
Review Board of General Hospital 'Agia Sophia' (protocol code, 24814; date of approval, October 30, 2017). Informed consent was obtained from all subjects involved in the study.

\section{Patient consent for publication}

Not applicable.

\section{Competing interests}

The authors declare that they have no competing interests.

\section{References}

1. Walani SR: Global burden of preterm birth. Int J Gynaecol Obstet 150: 31-33, 2020.

2. Markopoulou P, Papanikolaou E, Analytis A, Zoumakis E and Siahanidou T: Preterm birth as a risk factor for metabolic syndrome and cardiovascular disease in adult life: A systematic review and meta-analysis. J Pediatr 210: 69-80.e5, 2019.

3. Parets SE, Bedient CE, Menon R and Smith AK: Preterm birth and its long-term effects: Methylation to mechanisms. Biology (Basel) 3: 498-513, 2014.

4. Lyons KE, Ryan CA, Dempsey EM, Ross RP and Stanton C: Breast milk, a source of beneficial microbes and associated benefits for infant health. Nutrients 12: 1039, 2020

5. ESPGHAN Committee on Nutrition, Agostoni C, Braegger C, Decsi T, Kolacek S, Koletzko B, Michaelsen KF, Mihatsch W, Moreno LA, Puntis J, et al: Breast-feeding: A commentary by the ESPGHAN committee on nutrition. J Pediatr Gastroenterol Nutr 49: 112-125, 2009.

6. Kim KU, Kim WH, Jeong CH, Yi DY and Min H: More than nutrition: Therapeutic potential of breast milk-derived exosomes in cancer. Int J Mol Sci 21: 7327, 2020.

7. Cheshmeh S, Nachvak SM, Rezvani N and Saber A: Effects of breastfeeding and formula feeding on the expression level of FTO, CPT1A and PPAR- $\alpha$ genes in healthy infants. Diabetes Metab Syndr Obes 13: 2227-2237, 2020.

8. Horta BL, Victora CG, França GV, Hartwig FP, Ong KK, Rolfe EL, Magalhães EI, Lima NP and Barros FC: Breastfeeding moderates FTO related adiposity: A birth cohort study with 30 years of follow-up. Sci Rep 8: 2530, 2018.

9. Melnik B and Schmitz G: Milk's role as an epigenetic regulator in health and disease. Diseases 5: 12, 2017.

10. Boix-Amorós A, Collado MC, Van't Land B, Calvert A, le Doare K, Garssen J, Hanna H, Khaleva E, Peroni DG, Geddes DT, et al: Reviewing the evidence on breast milk composition and immunological outcomes. Nutr Rev 77: 541-556, 2019.

11. Seferovic MD, Mohammad M, Pace RM, Engevik M, Versalovic J, Bode L, Haymond M and Aagaard KM: Maternal diet alters human milk oligosaccharide composition with implications for the milk metagenome. Sci Rep 10: 22092, 2020.

12. Karlsson O, Rodosthenous RS, Jara C, Brennan KJ, Wright RO, Baccarelli AA and Wright RJ: Detection of long non-coding RNAs in human breastmilk extracellular vesicles: Implications for early child development. Epigenetics 11: 721-729, 2019.

13. Kim SY and Yi DY: Components of human breast milk: From macronutrient to microbiome and microRNA. Clin Exp Pediatr 63: 301-309, 2020.

14. Kalluri R and LeBleu VS: The biology, function, and biomedical applications of exosomes. Science 367: eaau6977, 2020.

15. van Niel G, D'Angelo G and Raposo G: Shedding light on the cell biology of extracellular vesicles. Nat Rev Mol Cell Biol 19: 213-228, 2018.

16. Soares Martins T, Catita J, Martins Rosa I, A B da Cruz E Silva O and Henriques AG: Exosome isolation from distinct biofluids using precipitation and column-based approaches. PLoS One 13: e0198820, 2018.

17. Vlachakis D, Mitsis T, Nicolaides N, Efthimiadou A, Giannakakis A, Bacopoulou F and Chrousos GP: Functions, pathophysiology and current insights of exosomal endocrinology (Review). Mol Med Rep 23: 26, 2021.

18. Madden JW: Human breast milk exosomes may protect against necrotizing enterocolitis in preterm infants. Pediatr Res 90: 244-245, 2021. 
19. Huang X, Yuan T, Tschannen M, Sun Z, Jacob H, Du M, Liang M, Dittmar RL, Liu Y, Liang M, et al: Characterization of human plasma-derived exosomal RNAs by deep sequencing. BMC Genomics 14: 319, 2013.

20. Valadi H, Ekström K, Bossios A, Sjöstrand M, Lee JJ and Lötvall JO: Exosome-mediated transfer of mRNAs and microRNAs is a novel mechanism of genetic exchange between cells. Nat Cell Biol 9: 654-659, 2007.

21. Statello L, Guo CJ, Chen LL and Huarte M: Gene regulation by long non-coding RNAs and its biological functions. Nat Rev Mol Cell Biol 22: 96-118, 2021.

22. Zhou Q, Li M, Wang X, Li Q, Wang T, Zhu Q, Zhou X, Wang X, Gao X and Li X: Immune-related microRNAs are abundant in breast milk exosomes. Int J Biol Sci 8: 118-123, 2021.

23. Bozack AK, Colicino E, Rodosthenous R, Bloomquist TR, Baccarelli AA, Wright RO, Wright RJ and Lee AG: Associations between maternal lifetime stressors and negative events in pregnancy and breast milk-derived extracellular vesicle microRNAs in the programming of intergenerational stress mechanisms (PRISM) pregnancy cohort. Epigenetics 16: 389-404, 2021.

24. Livak KJ and Schmittgen TD: Analysis of relative gene expression data using real-time quantitative PCR and the 2(-Delta Delta C(T)) method. Methods 25: 402-408, 2001

25. Galley JD and Besner GE: The therapeutic potential of breast milk-derived extracellular vesicles. Nutrients 12: 745, 2020.

26. Kahn S, Liao Y, Du X, Xu W, Li J and Lönnerdal B: Exosomal MicroRNAs in milk from mothers delivering preterm infants survive in vitro digestion and are taken up by human intestinal cells. Mol Nutr Food Res 62: e1701050, 2018.

27. Zhou Y, Yu Z, Wang X, Chen W, Liu Y, Zhang Y, Yin J and Han S: Exosomal circRNAs contribute to intestinal development via the VEGF signalling pathway in human term and preterm colostrum. Aging (Albany NY) 13: 11218-11233, 2021.

28. Gharesouran J, Taheri M, Sayad A, Ghafouri-Fard S, Mazdeh M and Omrani MD: The growth arrest-specific transcript 5 (GAS5) and nuclear receptor subfamily 3 group $\mathrm{C}$ member 1 (NR3C1): Novel markers involved in multiple sclerosis. Int J Mol Cell Med 7: 102-110, 2018

29. Ahmad I, Valverde A, Naqvi RA and Naqvi AR: Long non-coding RNAs RN7SK and GAS5 regulate macrophage polarization and innate immune responses. Front Immunol 11: 604981, 2020.

30. Mourtada-Maarabouni M, Hedge VL, Kirkham L, Farzaneh F and Williams GT: Growth arrest in human T-cells is controlled by the non-coding RNA growth-arrest-specific transcript 5 (GAS5). J Cell Sci 121: 939-946, 2008.

31. Jiang J, Hu H, Chen Q, Zhang Y, Chen W, Huang Q, Chen X, Li J and Zhong M: Long non-coding RNA SNHG29 regulates cell senescence via p53/p21 signaling in spontaneous preterm birth. Placenta 103: 64-71, 2021.

32. Yang Y,Pan JJ, Zhou XG, Zhou XY and Cheng R: Differentially expressed miRNAs in premature infants with retinopathy-a bioinformatics analysis. Int J Ophthalmol 11 773-779, 2018.
33. Ellis BC, Graham LD and Molloy PL: CRNDE, a long non-coding RNA responsive to insulin/IGF signaling, regulates genes involved in central metabolism. Biochim Biophys Acta 1843: 372-386, 2014

34. Huan J, Xing L, Lin Q, Xui H and Qin X: Long noncoding RNA CRNDE activates Wnt/ $\beta$-catenin signaling pathway through acting as a molecular sponge of microRNA-136 in human breast cancer. Am J Transl Res 9: 1977-1989, 2017.

35. Ellis BC, Molloy PL and Graham LD: CRNDE: A long non-coding RNA involved in CanceR, neurobiology, and development. Front Genet 3: 270, 2012.

36. Tichon A, Gil N, Lubelsky Y, Havkin Solomon T, Lemze D, Itzkovitz S, Stern-Ginossar N and Ulitsky I: A conserved abundant cytoplasmic long noncoding RNA modulates repression by pumilio proteins in human cells. Nat Commun 7: 12209, 2016.

37. Tichon A, Perry RBT, Stojic L and Ulitsky I: SAM68 is required for regulation of pumilio by the NORAD long noncoding RNA. Genes Dev 32: 70-78, 2018.

38. Kopp F, Elguindy MM, Yalvac ME, Zhang H, Chen B, Gillett FA, Lee S, Sivakumar S, Yu H, Xie Y, et al: Pumilio hyperactivity drives premature aging of Norad-deficient mice. Elife 8: e42650, 2019.

39. Lee S, Kopp F, Chang TC, Sataluri A, Chen B, Sivakumar S, Yu H, Xie Y and Mendell JT: Noncoding RNA NORAD regulates genomic stability by sequestering pumilio proteins. Cell 164: 69-80, 2016.

40. Munschauer M, Nguyen CT, Sirokman K, Hartigan CR, Hogstrom L, Engreitz JM, Ulirsch JC, Fulco CP, Subramanian V, Chen J, et al: The NORAD lncRNA assembles a topoisomerase complex critical for genome stability. Nature 561: 132-136, 2018.

41. Kawasaki N, Miwa T, Hokari S, Sakurai T, Ohmori K, Miyauchi K, Miyazono K and Koinuma D: Long noncoding RNA NORAD regulates transforming growth factor- $\beta$ signaling and epithelial-to-mesenchymal transition-like phenotype. Cancer Sci 109: 2211-2220, 2018.

42. Li H, Wang X, Wen C, Huo Z, Wang W, Zhan Q, Cheng D, Chen H, Deng X, Peng C and Shen B: Long noncoding RNA NORAD, a novel competing endogenous RNA, enhances the hypoxia-induced epithelial-mesenchymal transition to promote metastasis in pancreatic cancer. Mol Cancer 16: 169, 2017.

43. Martin A, Faes C, Debevec T, Rytz C, Millet G and Pialoux V: Preterm birth and oxidative stress: Effects of acute physical exercise and hypoxia physiological responses. Redox Biol 17: 315-322, 2018

44. Carney MC, Tarasiuk A, DiAngelo SL, Silveyra P, Podany A, Birch LL, Paul IM, Kelleher S and Hicks SD: Metabolism-related microRNAs in maternal breast milk are influenced by premature delivery. Pediatr Res 82: 226-236, 2017. International (CC BY-NC-ND 4.0) License. 